\title{
Computer-assisted analysis of caspases molecular evolution
}

\author{
Alexey Zamaraev \\ MSU, Moscow, Russia \\ a-zamaraev@yandex.ru
}

\author{
Gelina Kopeina \\ MSU, Moscow, Russia
}

\author{
Konstantin Gunbin \\ ICG SB RAS, Novosibirsk, Russia \\ NSU, Novosibirsk, Russia \\ genkvg@gmail.com
}

\begin{abstract}
The functions of any protein are driven by their chemical and physical properties, which, in turn, are determined by steric and physico-chemical folding requirements. Therefore, it is expected that replacement of the amino acid tightly interacting with a large number of other amino acids is related to changes in the context of interactions in the protein globule. Recent studies of the protein evolution revealed various signatures of substitution asymmetry and heterotachy. Here, based on reconstruction of ancestral libraries we analyze the substitution asymmetry in the molecular evolution of caspases protein family characterized by huge number of molecular functions.
\end{abstract}

Keywords - caspases, molecular evolution, substitution asymmetry, ancestral libraries

\section{Motivation and Aim}

Up to now, the vast majority of available procedures of reconstruction of ancestral sequences are based on the symmetric single (applied to all protein sites) matrix of amino acid substitution rates. It seems obvious that new software tools for ancestral protein reconstruction, taking into account substitution limitations from the 3D protein structure and from the stability of its folding (for example, ProtASR), should be most useful. However, unfortunately, the experimentally resolved $3 \mathrm{D}$ protein structures are still lacking. Another way for substitution asymmetry accounting in the ancestral protein reconstruction is the construction of ancestral libraries [1]. To make the ancestor libraries accurate enough, it has recently been proposed to use the AltAll reconstruction approach. This approach combines all possible alternative states introduced into one protein, and then characterizes this protein with a set of these states $[2,3]$. It has been shown that this approach significantly improves the imperfection of individual ancestral sequences reconstructed by Bayesian approach.

\section{Methods}

Multiple alignment was done using PROMALS. The best reversible and symmetric models of amino acid substitution rates were selected by IQTree v. 1.5.4 (in our case it is $\mathrm{C} 20+\mathrm{G} 4)$. We adjusted initial phylogenetic tree topology using Metazoa species tree from TimeTree DB by the TreeFix v. 1.1.10 software. After this, the branch lengths were reoptimized using IQTree v. 1.5.4 and the best reversible model of amino acid substitution rates. Bayesian sampling of ancestral sequences in each internal tree node was carried out using PhyloBayes v. 4.1, the CAT model [4], and 6 rate categories of sites. In order to construct complete and truncated (using our modified approach, called 'AltAll * N') libraries of ancestral sequences we used AltAll * N' procedure. Our procedure 'AltAll $* \mathrm{~N}$ ' is an iterative rewriting of all probable (with a posteriori probability $>0.1$ ) alternative states in a consensus ancestral sequence of given tree internal node. For example, if there are 3 alternative states in site $\mathrm{A}$ and 4 alternative states in site B of ancestral sequence (node) $\mathrm{X}$, then we must rewrite this ancestral sequence 4 times to get 4 alternative ancestors: a) a sequence consisting of the best states in A- and B-sites, b) a sequence with the second most probable states of sites $\mathrm{A}$ and $\mathrm{B}, \mathrm{c})$ a sequence with the third probable states of sites $\mathrm{A}$ and $\mathrm{B}$, and $\mathrm{d}$ ) a sequence with the third probable state in sites A and with the fourth probable in site B.

Substitution asymmetry during protein evolution was detected by analyzing deviations in the protein evolution rates from the reversible and symmetric model of amino acid substitution rates on each of the branches of the protein tree (1) and by a comparative analysis of the lengths of the internal tree branches taking into account the structure of the protein (2). (1) In order to solve first task, we: a) reconstructed a proteinspecific, time-reversible model of amino acid substitution rates using Modelestimator software; b) for each possible amino acid replacement on each internal node of the tree, we calculated $\mathrm{d}=\mathrm{PPa} * \mathrm{PPb} * 2 * \mathrm{NC}$, where $\mathrm{PPa}$ and $\mathrm{PPb}$ are the posterior probabilities of amino acids $a$ and $b, a \neq b$, $\mathrm{NC}=1 /\left(1+\mathrm{e}^{\wedge}(200 * \mathrm{RFab})\right), \quad \mathrm{RFab}$ is the relative rate of $\mathrm{ab}$ substitution in the time-reversible model of amino acid substitution rates; c) summed d values along all sites in each internal tree node and calculated the natural logarithms of these sums $\left(\ln \left(\sum \mathrm{d}\right)\right)$; d) in order to identify branches with a maximum $\ln \left(\sum \mathrm{d}\right)$, we conducted nonparametric comparison of these values over the entire tree. (2) In order to solve second task, we used truncated 'AltAll * N' libraries, particularly we: a) for each alternative ancestral sequence in each internal tree node, using the RaptorX_Property Fast software system, we assigned secondary structures to each amino acids, derived amino acid solvent accessibilities, and predicted disorder for each amino acid residue; b) calculated the frequency of changes of these measures between all alternative ancestral sequences of neighboring nodes residing on each internal tree branch; c) in order to identify branches with maximum structural changes, conducted nonparametric comparison of the abovementioned frequencies of changes across the entire tree.

\section{Results}

We extracted and verified 1565 Deuterostomia caspase proteins from NCBI GenBank. After that, we processed this protein set as described above.

One of the most promising observation we made is the finding of strict substitution asymmetry in the diversification of lower rodents, characterized by higher longevity comparing to higher rodents (Fig 1). The same evolutionary event is characteristic for caspase 7 in Xenopus (Fig 2). 


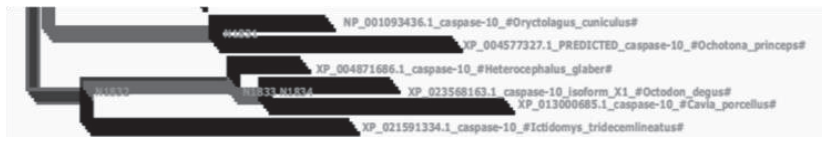

Fig. 1. Selected part of caspases phylogenetic tree, containing caspase 10 of lower rodents. Each branch composed of 5 lines, corresponding to (from down to up) changes in amino acid disorder, protein secondary structures (3 and 8 types of secondary structure), amino acid solvent accessibilities, and deviations from the reversible and symmetric mode of amino acid substitution. Black lines show terminal branches, light grey indicates evolution without significant deviations, while dark grey indicates significant deviations from expectations.

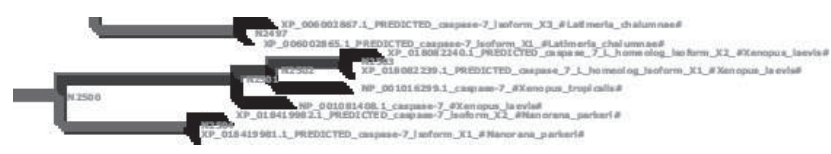

Fig. 2. Selected part of caspases phylogenetic tree, containing caspase 7 of Xenopus. Same designations as in Fig. 1.

It is of interest, that such evolutionary events are characteristic for the divergences of large protein clades tightly related with origination of large taxonomical groups, for example, avian caspase 6 , mammalian caspase 13 , etc.

\section{ACKNOWLEDGMENT}

This work supported by the grant from the RSF \# 19-1500125. BZ is supported by the Swedish (190345) and Stockholm (181301) Cancer Societies.

\section{REFERENCES}

[1] Gumulya Y, Gillam EM. (2017) Exploring the past and the future of protein evolution with ancestral sequence reconstruction: the 'retro'approach to protein engineering. Biochem J. 474(1):1-9.

[2] Anderson DP, et al. (2016) Evolution of an ancient protein function involved in organized multicellularity in animals. elife. 5:e10147.

[3] Eick GN, et al. (2017) Robustness of reconstructed ancestral protein functions to statistical uncertainty. Mol Biol Evol. 34(2):247-61.

[4] Quang le S, Gascuel O, Lartillot N. (2008) Empirical profile mixture models for phylogenetic reconstruction. Bioinformatics. 24(20):23172323. 